\title{
A Comparison of Higher-Order Difference Methods in the Solution of Beam-Vibration Problems
}

\author{
by DONALD T. GREENWOOD \\ Department of Aeronautical and Astronautical Engineering \\ University of Michigan \\ Ann Arbor, Michigan
}

Reprinted from the IEEE Transactions on Electronic Computers by permission of the copyright owner.

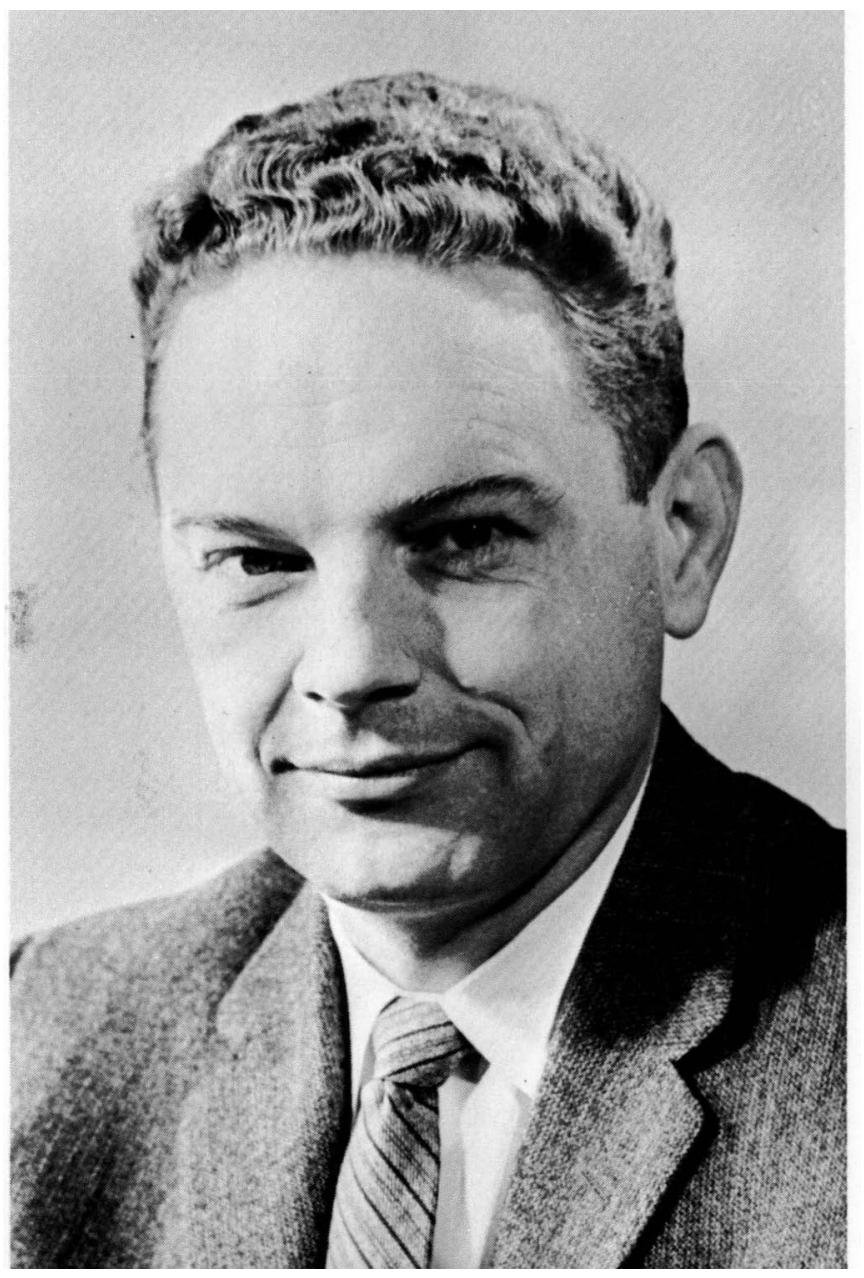

DONALD T. GREENWOOD was born in Clarkdale, Arizona, in 1923. He received his B.S. in mechanical engineering, his M.S. in physics, and his Ph.D. in electrical engineering, all from the California Institute of Technology in Pasadena.

From 1951 to 1956 he was in charge of analog computation at Lockheed Aircraft Corporation in Burbank, California. During '54-'55 he was also lecturer in Electrical Engineering at the University of Southern California. Since 1956 he has been at the University of Michigan, where he is Professor of Instrumentation. $\mathrm{He}$ is currently working in the area of computer solutions of dynamics problems associated with space vehicles.

$\mathrm{He}$ is a member of Tau Beta $\mathrm{Pi}$ and Sigma Xi.

\section{SUMMARY}

Several higher-order difference methods are investigated and compared for the problem of finding the natural frequencies of the lateral vibration of a beam. All of the methods considered are applicable to either digital or analog computers, although particular reference is made to the analog computer.

The methods considered in most detail use the same basic central difference approximation, the variations occurring in the method of representing boundary conditions. Three higher-order approaches to the problem of boundary conditions are presented. They are 1) the use of one-sided differences of fourth order, 2) the use of symmetry assumptions, and 3) the passive-circuit approach. Each method is shown to have its advantages, the final choice depending upon the particular requirements of the problem.

Results are presented in the form of curves of percentage mode-frequency error vs number of cells for the various approximation methods.

\section{INTRODUCTION}

Finite difference methods have been in use for quite some time in the approximate solution of partial differential equations. In particular, Howe and Haneman, ${ }^{1}$ McCann and MacNeal, ${ }^{2}$ and others have applied finite difference techniques to the analog computer solution of beam-vibration problems. These investigators have used simple central difference approximations to the spatial derivatives, these approximations having an error of order $h^{2}$, where $h$ is the cell size.

Fisher, ${ }^{3,4}$ has suggested the use of higher-order differences in the analog computer solution of partial differential equations and has given an indication of the accuracy and stability characteristics that might be expected. This approach looks particularly attrac- 
tive for use with electronic differential analyzers, because essentially the same number of amplifiers are required as for the simpler approximation. The added accuracy is obtained by using more inputs per amplifier. Similarly, in the digital computer formulation of the eigenvalue problem, the complexity of the matrices is increased, but their size and the nature of the computation is unchanged.

\section{The Beam Equations}

The partial differential equation describing the lateral motion of a beam can be written as

$$
\frac{\partial^{2}}{\partial \bar{x}^{2}}\left(E I \frac{\partial^{2} \bar{y}}{\partial \bar{x}^{2}}\right)+\rho \frac{\partial^{2} \bar{y}}{\partial t^{2}}=f(\bar{x}, \bar{t}),
$$

where

$$
\begin{aligned}
\bar{x} & =\text { position along beam } \\
\bar{y} & =\text { lateral deflection } \\
\vec{t} & =\text { time } \\
E I & =\text { bending stiffness } \\
\rho & =\text { mass per unit length } \\
t & =\text { lateral force per unit length. }
\end{aligned}
$$

For the case of an unforced uniform beam, one can nondimensionalize the equation to put it in the simpler form

$$
\frac{\partial^{4} y}{\partial x^{2}}+\frac{\partial^{2} y}{\partial t^{2}}=0
$$

Equivalently, one can write the four equations

$$
\begin{gathered}
\theta=\frac{\partial y}{\partial x} \\
M=\frac{\partial \theta}{\partial x} \\
V=\frac{\partial M}{\partial x} \\
\frac{\partial^{2} y}{\partial t^{2}}=-\frac{\partial V}{\partial x},
\end{gathered}
$$

where $\theta, M$, and $V$ are the slope, bending moment, and shear force, respectively, in the dimensionless system.

It should be noted that the equations have been simplified principally for ease of presentation. The analog computer circuits presented are valid for obtaining transient solutions with arbitrary forcing functions and for nonuniform beams.

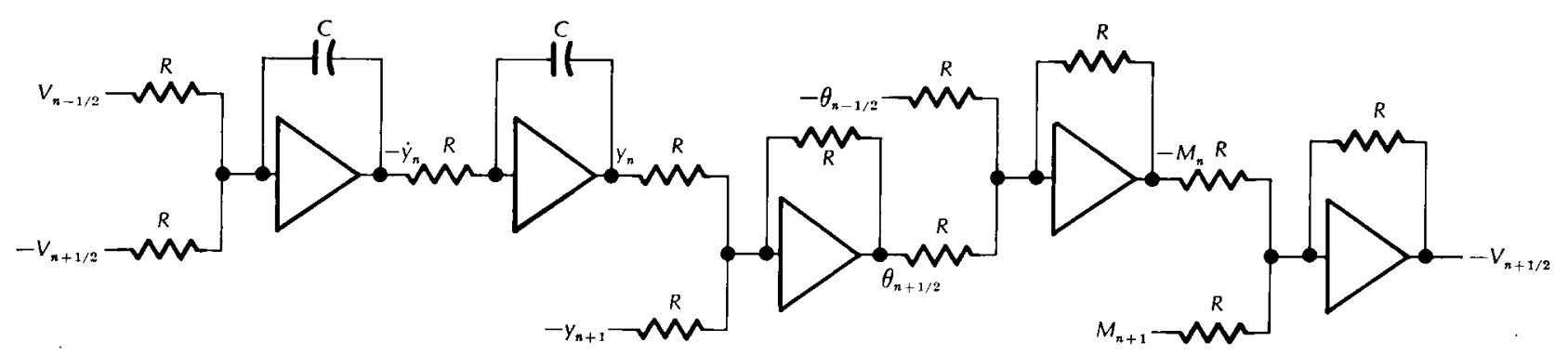

Figure 1 - The Analog Computer Circuit for a Typical Beam Cell Using Approximations of Order $h^{2}$ to Four First-Order

Differential Equations

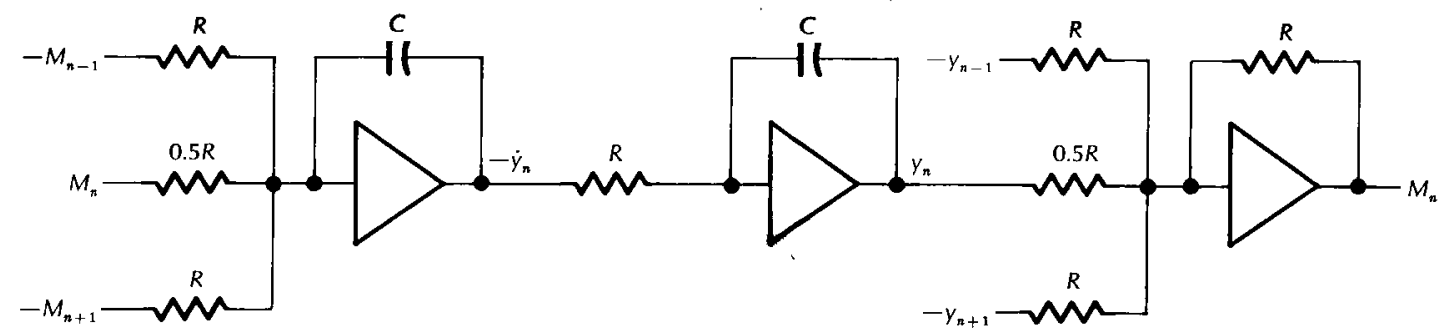

Figure 2 - The Analog Computer Circuit for a Typical Beam Cell Using Approximations of Order $h^{2}$ to Two Second-Order Differential Equations 


\section{Approximations of Order $h^{2}$}

In order to obtain a better appreciation of the diferences between the ordinary and higher-order approaches to the formulation of the problem, consider first the use of simple central difference equations in which the derivative is approximated at the midpoint of the input stations. The basic approximation is ${ }^{3}$

$$
\left.\frac{\partial y}{\partial x}\right|_{n+1 / 2} \cong \frac{-y_{n}+y_{n+1}}{h}-\left.\frac{h^{2}}{24} \frac{\partial^{3} y}{\partial x^{33}}\right|_{n+1 / 2}{ }^{\prime}
$$

where the last term on the right is the principal error term. For reasons of accuracy we use staggered stations, i.e., we calculate $y$ and $M$ at integer stations and calculate $\theta$ and $V$ at half-integer stations. The difference equations corresponding to (3) through (6) are

$$
\begin{aligned}
\theta_{n+1 / 2} & =\frac{1}{h}\left(-y_{n}+y_{n+1}\right) \\
M_{n} & =\frac{1}{h}\left(-\theta_{n-1 / 2}+\theta_{n+1 / 2}\right) \\
V_{n+1 / 2} & =\frac{1}{h}\left(-M_{n}+M_{n+1}\right) \\
\ddot{y}_{n} & =\frac{1}{h}\left(V_{n-1 / 2}-V_{n+1 / 2}\right)
\end{aligned}
$$

where a dot over a quantity denotes differentiation with respect to time. Thus, each partial differential equation has been replaced by a set of ordinary differential equations in which time is the independent variable.

The analog computer circuit for solving the above equations requires five amplifiers per cell with up to two inputs per amplifier, as is shown in Figure 1. If a solution for $\theta$ and $V$ is not required, one can simplify the circuit to three amplifiers per cell with up to three inputs per amplifier as shown in Figure 2. The circuits are drawn for $h=1$.

For the case of these approximations, the establishment of boundary conditions to order $h^{2}$ is quite straightforward. For example, a built-in end at station $n+1 / 2$ implies that

$$
y_{n}=y_{n+1}=0 \text {, }
$$

where one assumes a virtual beam beyond the end of the actual beam. Similarly, for a free end at station $n+1 / 2$ we obtain

$$
M_{n}=M_{n+1}=0 \text {. }
$$

One can proceed to calculate the percentage error as a function of the number of cells $N$ for various end conditions. ${ }^{5,6}$ These results are given for built-in and cantilever beams in Graphs 1 and 2.

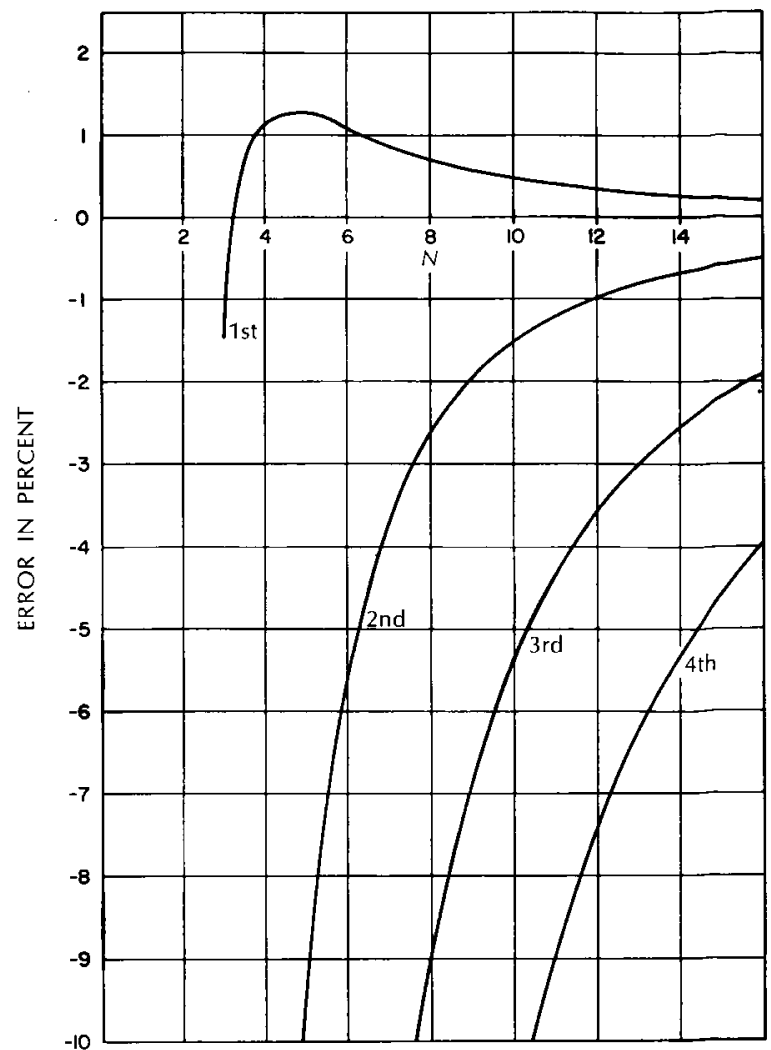

Graph 1 - Per Cent Error in Mode Frequency for a Built-in Beam, $h 2$ Approximation

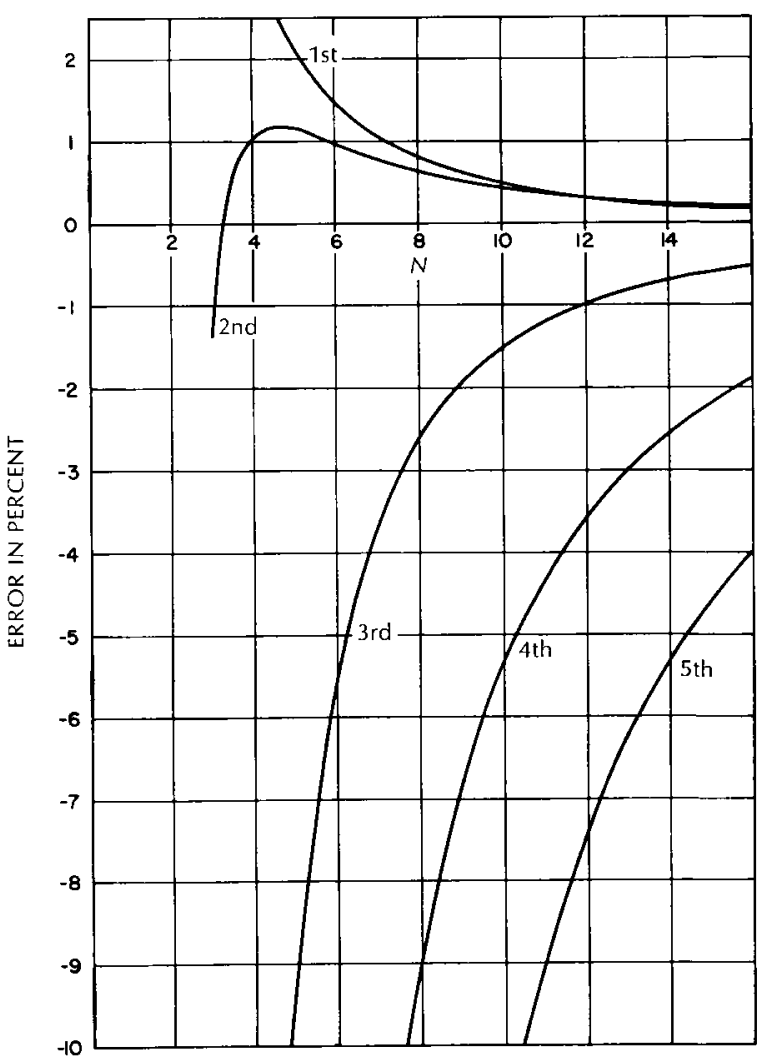

Graph $2-$ Per Cent Error in Mode Frequency for a Cantilever Beam, $h^{2}$ Approximation 


\section{Approximations of Order $h^{4}$}

The basic higher-order difference approximation to be used in this paper is

$$
\begin{aligned}
\left.\frac{\partial y}{\partial x}\right|_{n+1 / 2} \cong \frac{1}{24 h}-\left(y_{n-1}-27 y_{n}\right. & \left.+27 y_{n+1}-y_{n+2}\right) \\
& +\left.\frac{3 h^{4}}{640} \frac{\partial^{5} y}{\partial x^{5}}\right|_{n+1 / 2} .
\end{aligned}
$$

Thus, we again use staggered stations and obtain the higher-order equivalent of (8) through (11).

$$
\begin{gathered}
\theta_{n+1 / 2}=\frac{1}{24 h}\left(y_{n-1}-27 y_{n}+27 y_{n+1}-y_{n+2}\right) \\
M_{n}=\frac{1}{24 h}\left(\theta_{n-3 / 2}-27 \theta_{n-1 / 2}+27 \theta_{n+1 / 2}-\theta_{n+3 / 2}\right) \\
V_{n+1 / 2}=\frac{1}{24 h}\left(M_{n-1}-27 M_{n}+27 M_{n+1}-M_{n+2}\right) \\
\ddot{y}_{n}=\frac{1}{24 h}\left(-V_{n-3 / 2}+27 V_{n-1 / 2}-27 V_{n+1 / 2}+V_{n+3 / 2}\right) .
\end{gathered}
$$

The analog computer circuit for a typical cell is shown in Figure 3. Note that five amplifiers are required with up to four inputs for each amplifier.

In this case the reasons were particularly strong for not calculating all variables at each station. For example if we had used the approximation

$$
\begin{array}{r}
\left.\frac{\partial y}{\partial x}\right|_{n} \cong \frac{1}{12 h}\left(y_{n-2}-8 y_{n-1}+8 y_{n+1}-y_{n+2}\right) \\
+\left.\frac{h^{4}}{30} \frac{\partial^{5} y}{\partial x^{5}}\right|_{n}
\end{array}
$$

nine amplifiers per cell would have been required, and the principal error term would have been approximately seven times larger.

\section{BOUNDARY CONDITION METHODS}

Equations (15)-(18) can be used in a straightforward manner for stations well away from the ends. However, as an end is approached, some of the needed stations lie beyond the end, and further assumptions must be made. Three methods of handling the problem of boundary conditions will be given and comparisons made of the accuracy and other important features of each method.

\section{The $h^{4}$ Approximation}

A natural approach to the problem of properly applying boundary conditions is to use one-sided differences of order $h^{4}$ at points where the usual central differences cannot be used. It will be necessary to use up to five stations to obtain the necessary accuracy, depending upon the end conditions of the function in question.

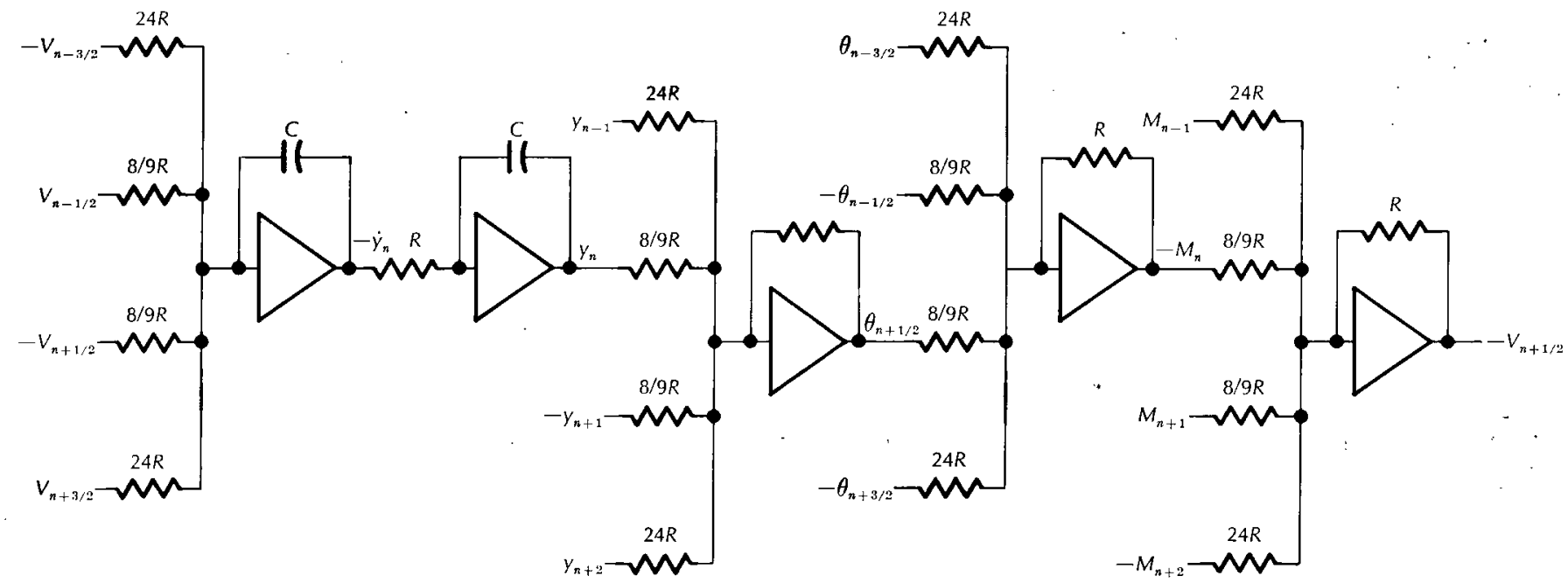

Figure 3-Analog Computer Circuit for a Typical Cell Using

Staggered Stations and a Central Difference Approximation of Order $h^{4}$ 
Consider first the case of a beam that is built-in at its left end, which occurs at station $1 / 2$ (see Figure 4).

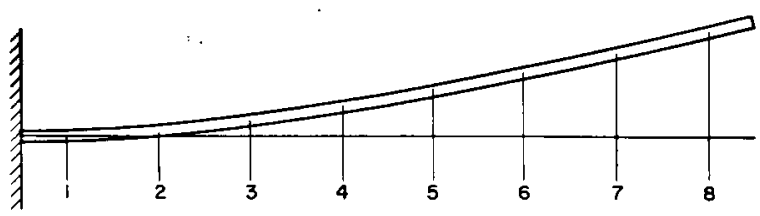

Figure 4-Cantilever Beam Showing Station Locations

Assuming that $y_{1 / 2}$ and $\theta_{1 / 2}$ are zero, one can use the approximation

$\theta_{3 / 2} \cong \frac{1}{225 h}\left(-225 y_{1}+250 y_{2}-9 y_{3}\right)+\left.\frac{1}{240} h^{4} \frac{\partial^{5} y}{\partial x^{5}}\right|_{3 / 2}$

Also, it can be shown that

$$
y_{1} \cong \frac{2}{9} y_{2}-\frac{1}{25} y_{3}+\left.\frac{29}{2880} h^{6} \frac{\partial^{6} y}{\partial x^{6}}\right|_{1 / 2}
$$

for the case of a uniform beam. Eliminating $y_{1}$ and using (15) directly at the other stations, the matrix equation

$$
\left\{\begin{array}{c}
\theta_{3 / 2} \\
\theta_{5 / 2} \\
\theta_{\tau / 2} \\
\vdots
\end{array}\right\}=\frac{1}{5400 h}\left[\begin{array}{rcccc}
4800 & 0 & 0 & 0 & \cdots \\
-6025 & 6066 & -225 & 0 \\
225 & -6075 & 6075 & -225 & \cdots \\
\vdots & & & \vdots
\end{array}\right]\left\{\begin{array}{l}
y_{2} \\
y_{3} \\
y_{4} \\
\vdots \\
\vdots
\end{array}\right\}
$$

is obtained, where terms of order $h^{4}$ or higher have been omitted.

Similarly, using the approximation

$$
\begin{array}{r}
M_{1} \cong \frac{1}{24 h}\left(-22 \theta_{1 / 2}+17 \theta_{3 / 2}+9 \theta_{5 / 2}-5 \theta_{7 / 2}+\theta_{9 / 2}\right) \\
-\left.\frac{71}{1920} h^{4} \frac{\partial^{5} \theta}{\partial x^{5}}\right|_{1}
\end{array}
$$

and noting that $\theta_{1 / 2}$ is zero, we obtain

$\left\{\begin{array}{c}M_{1} \\ M_{2} \\ M_{3} \\ \vdots\end{array}\right\}=\frac{1}{24 h}\left[\begin{array}{rrrrl}17 & 9 & -5 & 1 & 0 \\ -27 & 27 & -1 & 0 & 0 \\ 1 & -27 & 27 & -1 & 0 \cdots \\ \vdots & & & & \vdots\end{array}\right]\left\{\begin{array}{c}\theta_{3 / 2} \\ \theta_{5 / 2} \\ \theta_{7 / 2} \\ \vdots\end{array}\right\}$

Also, using the approximations

$$
\begin{aligned}
V_{1 / 2} \cong \frac{1}{24 h}\left(-93 M_{1}\right. & +229 M_{2}-225 M_{3}+111 M_{4} \\
& \left.-22 M_{5}\right)+\left.\frac{563}{640} h^{4} \frac{\partial^{5} M}{\partial x^{5}}\right|_{1 / 2}
\end{aligned}
$$

and

$$
\begin{array}{r}
V_{3 / 2}=\frac{1}{24 h}\left(-22 M_{1}+17 M_{2}+9 M_{3}-5 M_{4}+M_{5}\right) \\
-\left.\frac{71}{1920} h^{4} \frac{\partial^{5} M}{\partial x^{5}}\right|_{3 / 2} .
\end{array}
$$

We obtain

$$
\left\{\begin{array}{c}
V_{1 / 2} \\
V_{3 / 2} \\
V_{5 / 2} \\
\vdots
\end{array}\right\}=\frac{1}{24 h}\left[\begin{array}{rrrrr}
-93 & 229 & -225 & 111 & -22 \cdots \\
-22 & 17 & 9 & -5 & 1 \\
1 & -27 & 27 & -1 & 0 \cdots \\
\vdots & & & & \vdots
\end{array}\right]\left\{\begin{array}{c}
M_{1} \\
M_{2} \\
M_{3} \\
\vdots \\
\vdots
\end{array}\right.
$$

Finally,

$$
\left\{\begin{array}{c}
\ddot{y}_{2} \\
\ddot{y}_{3} \\
\ddot{y}_{4} \\
\vdots
\end{array}\right\}=\frac{1}{24 h}\left[\begin{array}{rrrrc}
-1 & 27 & -27 & 1 & 0 \cdots \\
0 & -1 & 27 & -27 & 1 \\
0 & 0 & -1 & 27 & -27 \cdots \\
\vdots & & & & \vdots
\end{array}\right]\left\{\begin{array}{c}
V_{1 / 2} \\
V_{3 / 2} \\
V_{5 / 2} \\
\vdots \\
\vdots
\end{array}\right\}
$$

Equations (22), (24), (27), and (28) can be written in the form

$$
\begin{aligned}
\{\theta\} & =\left[B_{1}\right]\{y\} \\
\{M\} & =\left[B_{2}\right]\{\theta\} \\
\{V\} & =\left[B_{3}\right]\{M\} \\
\{\ddot{y}\} & =\left[B_{4}\right]\{V\}
\end{aligned}
$$

or

$$
\{\ddot{y}\}+[K]\{y\}=0,
$$

where the square $K$ matrix is

$$
[K]=-\left[B_{4}\right]\left[B_{3}\right]\left[B_{2}\right]\left[B_{1}\right] .
$$

Assuming solutions to (33) of the form

$$
y=A e^{j \lambda t} \quad(j=\sqrt{-1}),
$$

we obtain the determinantal equation

$$
\left|K-\lambda^{2} I\right|=0,
$$

where $l$ is the unit matrix. The roots of this equation are the natural frequencies in radians per second.

The representation of a free end using the $h^{4}$ approximation is very similar to the built-in case, except that the roles of $y$ and $M$ have been interchanged as well as those of $\theta$ and $V$. Thus we might think of $y$ and $M$ and also $\theta$ and $V$ as complementary quantities.

The result of calculations for the frequency errors of built-in and cantilever uniform beams is given as 


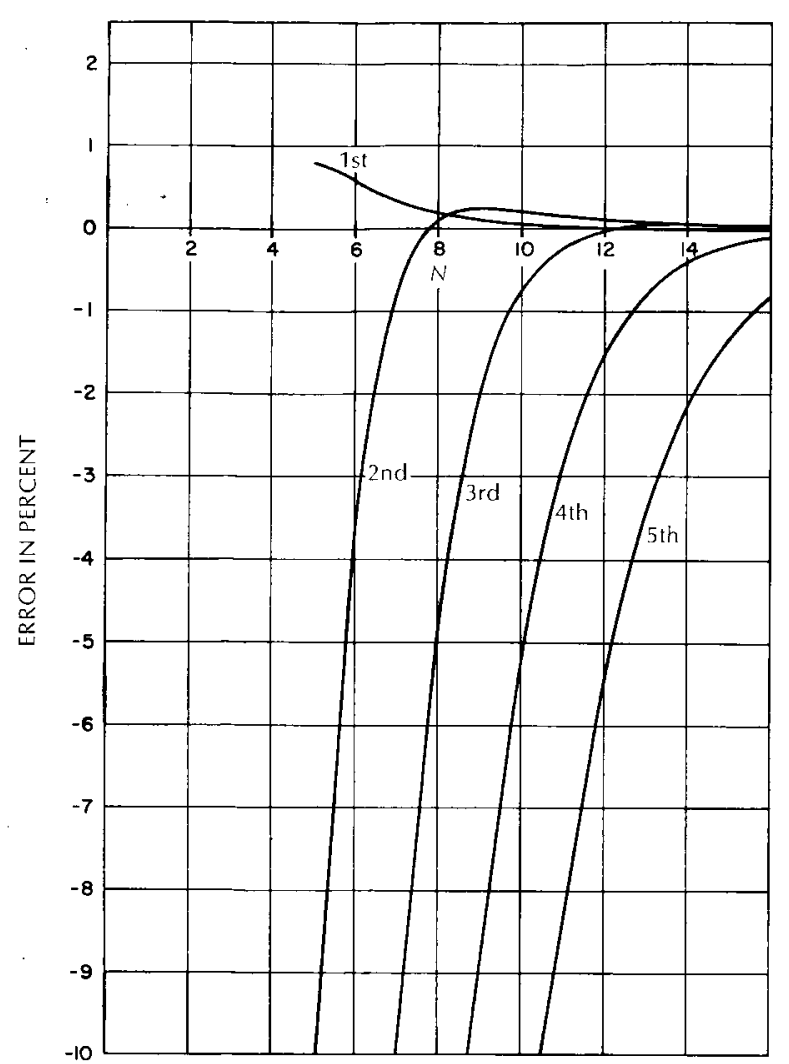

Graph 3 -Per Cent Error in Mode Frequency for a Built-in Beam, $h^{4}$ Approximation

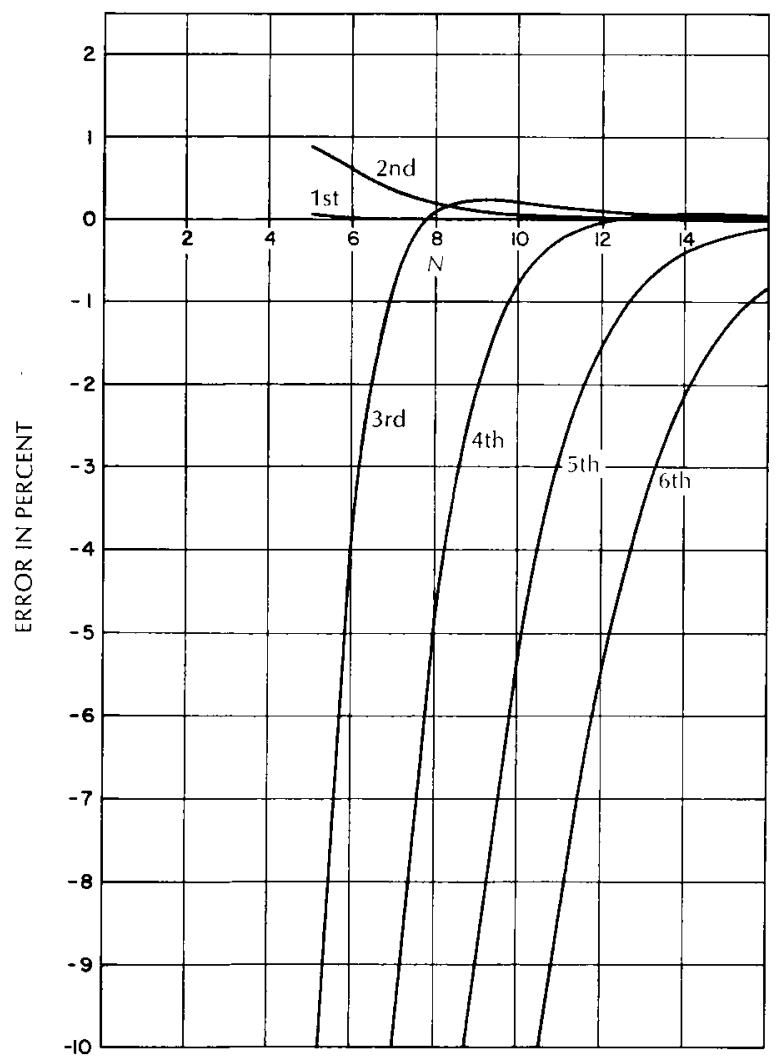

Graph 4-Per Cent Error in Mode Frequency for a Cantilever Beam, $h^{4}$ Approximation a function of the number of cells $N$ in Graphs 3 and 4. It should be noted that the error curve for the $(i+1)$ cantilever mode is nearly the same as that for mode $i$ of the built-in case, and the match is virtually exact for large $i$ and $N$. This applies to the other boundary condition approximations as well. Also note that the non-zero frequencies for a free beam are identical with those for a built-in beam.

The Use of Symmetry Assumptions

Another approach to the problem of representing boundary conditions is to use the basic approximation given by (14) at all stations, but make suitable assumptions concerning the extrapolation of the problem variables for virtual stations beyond the end of the beam.

For example, one might assume that the deflection curve has even symmetry about a built-in end. Successive differentiations would give alternating odd and even symmetry for $\theta, M$, and $V$. Also implied is a discontinuity in $V$ (or the slope of $M$ ) at the builtin end. Now suppose we interpret $\theta_{1 / 2}$ and $V_{1 / 2}$ to mean the values of slope and shear force at a point a very small distance from the wall and inside the actual beam. Then it will be seen that any extrapolation which includes a discontinuity will result in increased error. Therefore, we will always assume smooth extrapolations beyond the boundaries. The assumptions for built-in free ends are shown in Figure 5 . The rule followed in the extrapolations is that any curve which has zero slope at the boundary is continued assuming even symmetry. Any curve with non-zero slope is continued assuming odd symmetry, possibly with an offset such that there is no discontinuity.

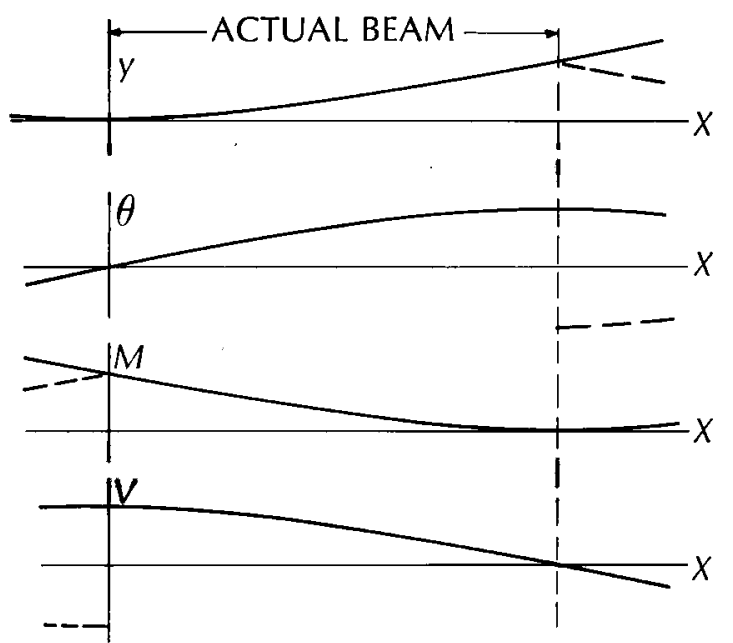

Figure 5-Symmetry Assumptions for a Cantilever Beam. Solid Lines Show Assumptions Used in Computations. Dashed Lines Indicate Deviations Giving a Physically Consistent Set of Assumptions 
The application of these assumptions is quite clear except for the case of the bending moment near a built-in end, or the deflection near a free end. For the case of a beam that is built-in at its left-hand end at station $1 / 2$, let us assume a variation in $M$ of the form

$$
M=b_{0}+b_{1} x+b_{3} x^{3}
$$

which has the required odd symmetry with an offset. One can solve for $b_{0}, b_{1}$, and $b_{3}$ and obtain the following equations giving values of $M$ in the virtual beam:

$$
\begin{aligned}
M_{0} & =(1 / 3)\left(7 M_{1}-5 M_{2}+M_{3}\right) \\
M_{-1} & =(1 / 3)\left(10 M_{1}-8 M_{2}+M_{3}\right) .
\end{aligned}
$$

Now we can write the equations of motion for a uniform beam that is built-in at the left end. Again, we use (21) to eliminate $y_{1}$ :

$$
\left\{\begin{array}{c}
\theta_{3 / 2} \\
\theta_{\overline{5} / 2} \\
\theta_{7 / 2} \\
\vdots
\end{array}\right\}=\frac{1}{5400 h}\left[\begin{array}{rrrc}
4775 & 9 & 0 & 0 \cdots \\
-6025 & 6066 & -225 & 0 \\
225 & -6075 & 6075 & -225 \cdots \\
\vdots & & & \vdots
\end{array}\right]\left\{\begin{array}{c}
y_{2} \\
y_{3} \\
y_{4} \\
\vdots \\
\vdots
\end{array}\right\}
$$

$$
\left\{\begin{array}{c}
M_{1} \\
M_{2} \\
M_{3} \\
\vdots
\end{array}\right\}=\frac{1}{24 h}\left[\begin{array}{rrrc}
26 & -1 & 0 & 0 \cdots \\
-27 & 27 & -1 & 0 \\
1 & -27 & 27 & -1 \\
\vdots & & & \vdots
\end{array}\right]\left\{\begin{array}{c}
\theta_{3 / 2} \\
\theta_{5 / 2} \\
\theta_{\tau / 2} \\
\vdots
\end{array}\right\}
$$

$$
\left\{\begin{array}{c}
V_{1 / 2} \\
V_{3 / 2} \\
V_{5 / 2} \\
\vdots
\end{array}\right\}=\frac{1}{72 h}\left[\begin{array}{rrrc}
-98 & 124 & -26 & 0 \cdots \\
-74 & 76 & -2 & 0 \\
3 & -81 & 81 & -3 \cdots \\
\vdots & & & \vdots
\end{array}\right]\left\{\begin{array}{c}
M_{1} \\
M_{2} \\
M_{3} \\
\vdots
\end{array}\right\}
$$

$$
\left\{\begin{array}{c}
\ddot{y}_{2} \\
\ddot{y}_{3} \\
\ddot{y}_{4} \\
\vdots
\end{array}\right\}=\frac{1}{24 h}\left[\begin{array}{rrrrr}
-1 & 27 & -27 & 1 & 0 \cdots \\
0 & -1 & 27 & -27 & 1 \\
0 & 0 & -1 & 27 & -27 \\
\vdots & & & & \vdots
\end{array}\right]\left\{\begin{array}{c}
V_{1 / 2} \\
V_{3 / 2} \\
V_{5 / 2} \\
\vdots
\end{array}\right\}
$$

From the above equations one can see that the analog computer circuit will never require more than four inputs per amplifier, whereas the $h^{4}$ approximation required up to five inputs per amplifier.

For any specific case, one can perform the matrix multiplications as in (34) and solve for the roots of (36). The frequency errors are plotted against the cell number $N$ for built-in and cantilever beams in Graphs 5 and 6 . Comparing these results with those ob-

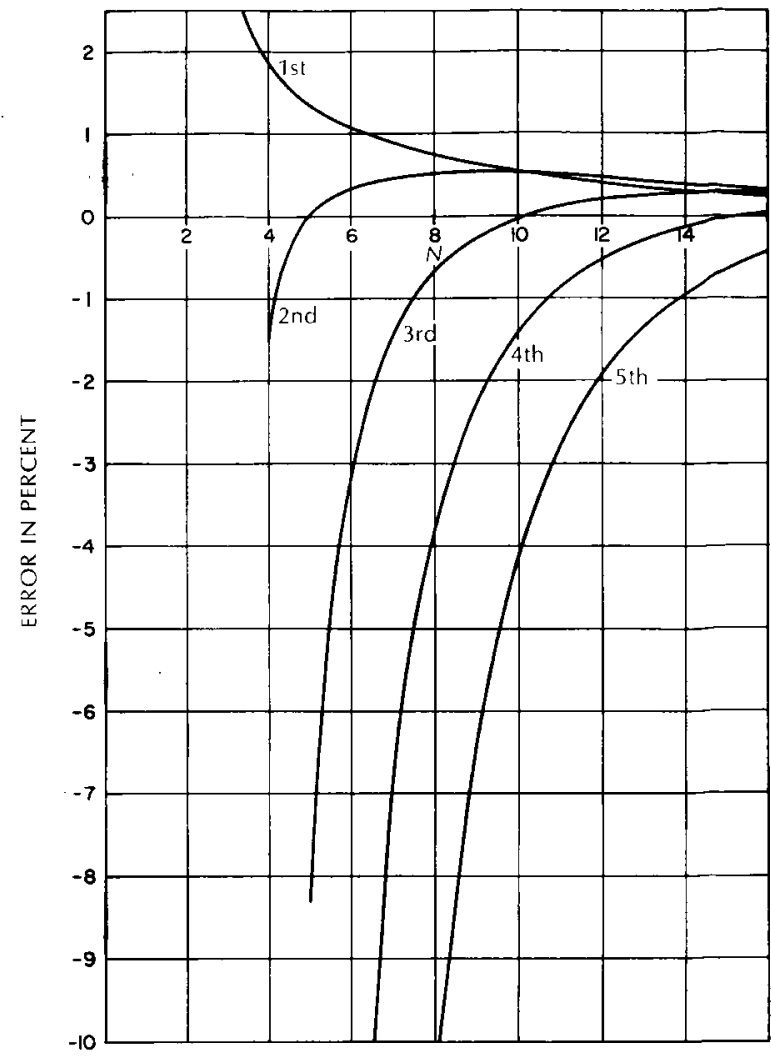

Graph 5-Per Cent Error in Mode Frequency for a Built-in Beam, Using Symmetry

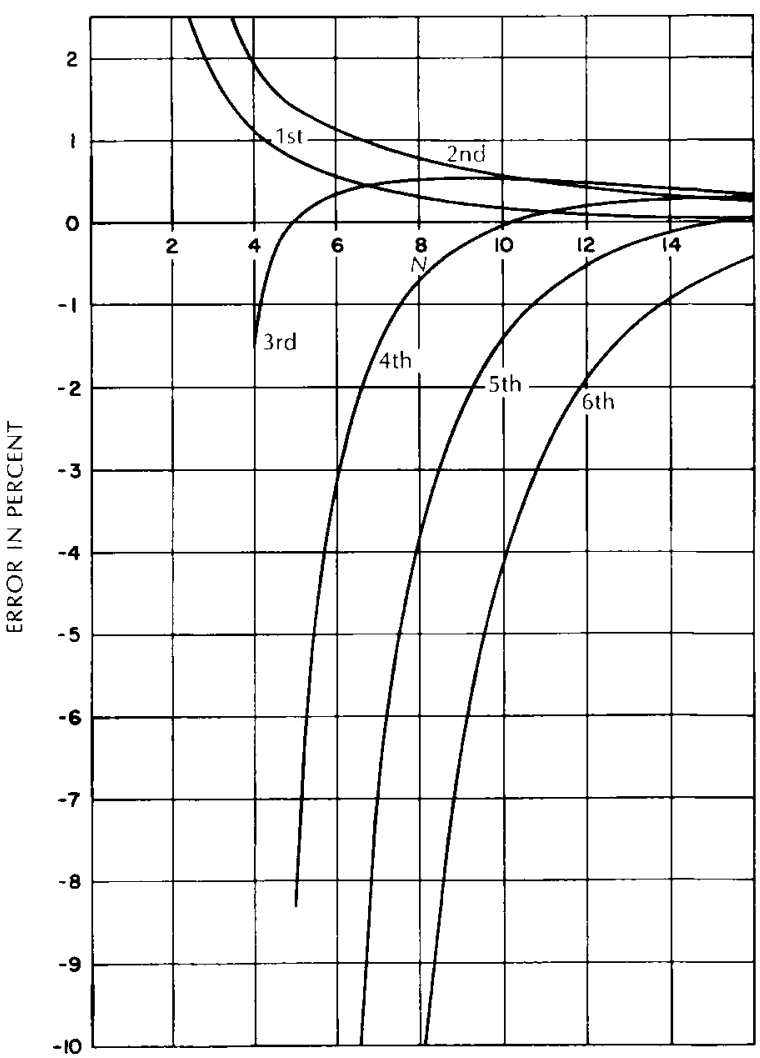

Graph 6-Per Cent Error in Mode Frequency for a Cantilever Beam, Using Symmetry 
tained previously, we find that the use of symmetry at the boundaries has improved the accuracy of the higher modes. More specifically, one can observe how large $N$ must be in order to obtain a given accuracy. For an accuracy of 1 per cent or better, it turns out that about $4 \frac{1}{2}$ cells per wavelength are required. By comparison, the $h^{4}$ approximation requires about 5 cells per wavelength and the $h^{2}$ approximation requires about 8 cells per wavelength.* On the other hand, if one is interested in only the lowest couple of modes, but with accuracies of the order of 0.25 per cent, then the $h^{4}$ approximation would be preferable. It should be noted, however, that component tolerance errors in analog computers (or round-off errors in digital computers) can be important in this accuracy range.

\section{The Passive Circuit Analogy}

Both higher-order methods of representing boundary conditions that have been discussed so far are non-physical in the sense that no passive linear sys-

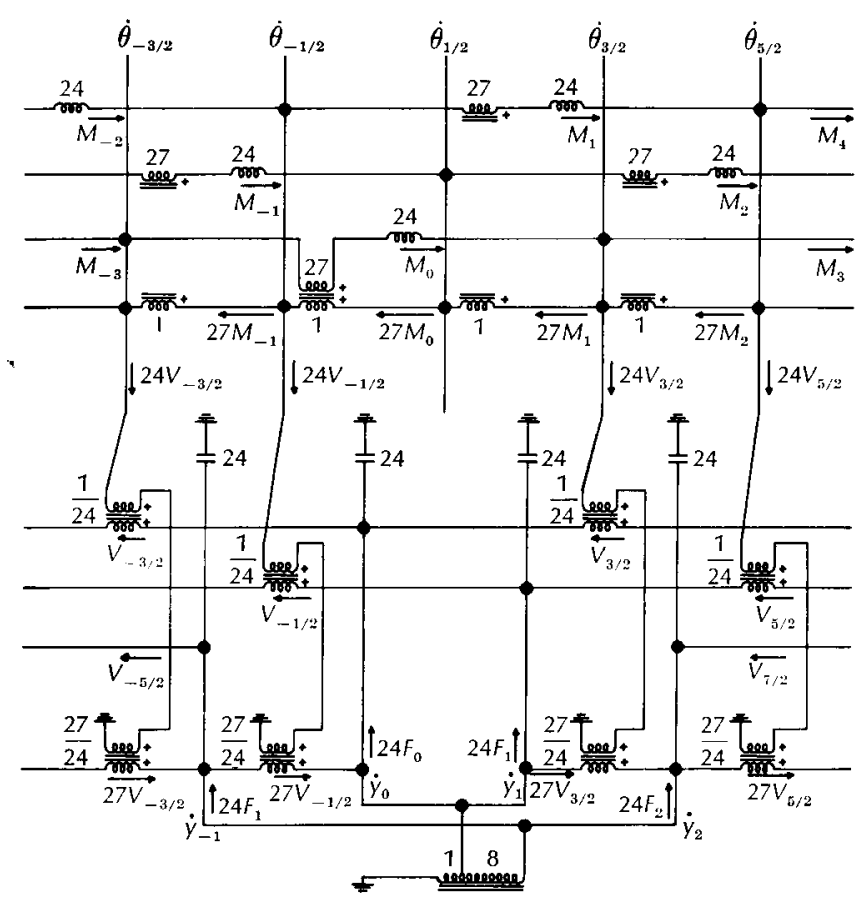

Figure 6-Passive Circuit Analogy for a Built-in End at Station $1 / 2$

\footnotetext{
* For the case of the higher modes and using large numbers of cells such that boundary conditions have little effect, the $h^{2}$ method requires 18 cells per wavelength and all $h^{4}$ methods require six cells per wavelength for 1 per cent frequency error.
}

tem can be found which is described exactly by the equations of motion. For many people, the lack of a physical model makes the problem more difficult conceptually. Furthermore, the stiffness matrix for the system

$$
[k]=[m][K],
$$

where $[m]$ is the mass matrix of the lumped system, turns out to be nonsymmetric, resulting in nonorthogonal modes. That is, the mass and stiffness matrices are not diagonalized by a transformation to modal coordinates.

While these shortcomings may not be great, they can be avoided by developing a physical model for the basic equations (15)-(18) and applying appropriate boundary conditions to this model. This has been done. The resulting passive-circuit analogy is shown in Figure 6 for the case of a beam that is builtin at station $1 / 2$.

In this passive-circuit analogy, shear forces and bending moments are represented by currents. The velocities $\dot{y}$ and $\dot{\theta}$ are represented by voltages. Masses are represented by capacitors and elastic properties by inductors. The transformer network provides the higher-order approximation to the spatial derivatives and also imposes constraints on the system. It may be seen that one capacitor, one inductor, and three transformers are required for a typical cell. The large number of transformers required makes it impractical to use this circuit directly in the solution of beamvibration problems. We will use it only as an aid in writing the equations of motion.

Now consider the equations of motion near a built-in end. Again we assume even symmetry for the deflection curve and odd symmetry for $\theta$. In contrast to the previous symmetry assumptions, however, $M$ and $V$ have even and odd symmetry, respectively. With these assumptions one can obtain suitable accuracy by using the constraint equation

$$
y_{1}=\frac{1}{9} y_{2}
$$

In accordance with the physics of the system, the forces of constraint (represented by currents $24 F_{1}$ and $24 F_{2}$ in Figure 6) are also included in the analysis.

Writing the equations of motion for the circuit corresponding to a beam with the left end built-in, one obtains

$$
\left\{\begin{array}{c}
\theta_{3 / 2} \\
\theta_{5 / 2} \\
\theta_{7 / 2} \\
\vdots
\end{array}\right\}=\frac{1}{216 h}\left[\begin{array}{rrrc}
217 & -9 & 0 & 0 \cdots \\
-242 & 243 & -9 & 0 \\
9 & -243 & 243 & -9 \cdots \\
\vdots & & & \vdots
\end{array}\right]\left\{\begin{array}{c}
y_{2} \\
y_{3} \\
y_{4} \\
\vdots \\
\vdots
\end{array}\right\}(46)
$$




$$
\left\{\begin{array}{c}
M_{1} \\
M_{2} \\
M_{3} \\
\vdots
\end{array}\right\}=\frac{1}{24 h}\left[\begin{array}{rrrc}
26 & -1 & 0 & 0 \\
-27 & 27 & -1 & 0 \\
1 & -27 & 27 & -1 \cdots \\
\vdots & & & \vdots
\end{array}\right]\left\{\begin{array}{c}
\theta_{3 / 2} \\
\theta_{5 / 2} \\
\theta_{7 / 2} \\
\vdots
\end{array}\right\}
$$

$$
\left\{\begin{array}{c}
V_{3 / 2} \\
V_{5 / 2} \\
V_{7 / 2} \\
\vdots
\end{array}\right\}=\frac{1}{24 h}\left[\begin{array}{rrrc}
-26 & 27 & -1 & 0 \cdots \\
1 & -27 & 27 & -1 \\
0 & 1 & -27 & 27 \\
\vdots & & & \vdots \\
\vdots & & & \vdots
\end{array}\right]\left\{\begin{array}{c}
M_{1} \\
M_{2} \\
M_{3} \\
\vdots
\end{array}\right\}
$$

$$
\left\{\begin{array}{c}
\ddot{y}_{2} \\
\ddot{y}_{3} \\
\ddot{y}_{4} \\
\vdots
\end{array}\right\}=\frac{1}{1968 h}\left[\begin{array}{rrrc}
1953 & -2178 & 81 & 0 \cdots \\
-82 & 2214 & -2214 & 82 \\
0 & -82 & 2214 & -2214 \cdots \\
\vdots & & . & \vdots
\end{array}\right\}\left\{\begin{array}{c}
V_{3 / 2} \\
V_{5 / 2} \\
V_{7 / 2} \\
\vdots
\end{array}\right\}
$$

The amount of analog computer equipment required in this case is essentially identical to that of the previous cases, but slight savings in amplifiers can be made near the boundaries, because $V$ at a built-in end (or $\theta$ at a free end) need not be generated. Also the signs are such that a few inverting amplifiers may be saved. No amplifier requires more than four inputs.

The error curves for the passive-circuit representation of built-in and cantilever beams are shown in Graphs 7 and 8 . It is seen that the error curves show more positive overshoot for low $N$ than in the previous case using symmetry assumptions and, for the most part, the calculated frequencies are slightly larger. However, one needs only about 4 cells per wavelength in this case to obtain 1 per cent accuracy. Furthermore, the $k$ (stiffness) matrix for the system is symmetric and the modes are orthogonal, thereby simplifying the detection of errors for digital computations.

\section{The Pinned Beam}

Error calculations have also been made for the case of a beam pinned at both ends. This implies that $y$ and $M$ are zero at the ends. In this case there is no problem of boundary condition representation, since one can assume odd symmetry for $y$ and $M$ and even symmetry for $\theta$ and $V$ at the boundary with perfect consistency. Thus any error is due to the fundamental approximation of (14).

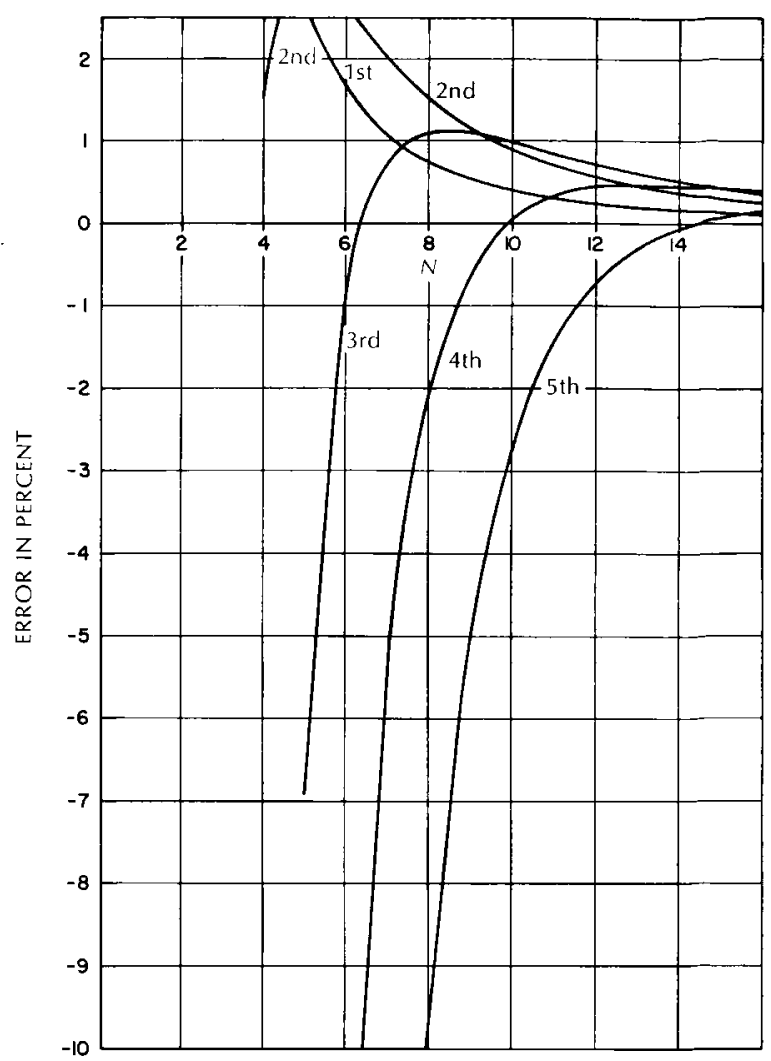

Graph 7 - Per Cent Error in Mode Frequency for a Built-in Beam, Passive Circuit

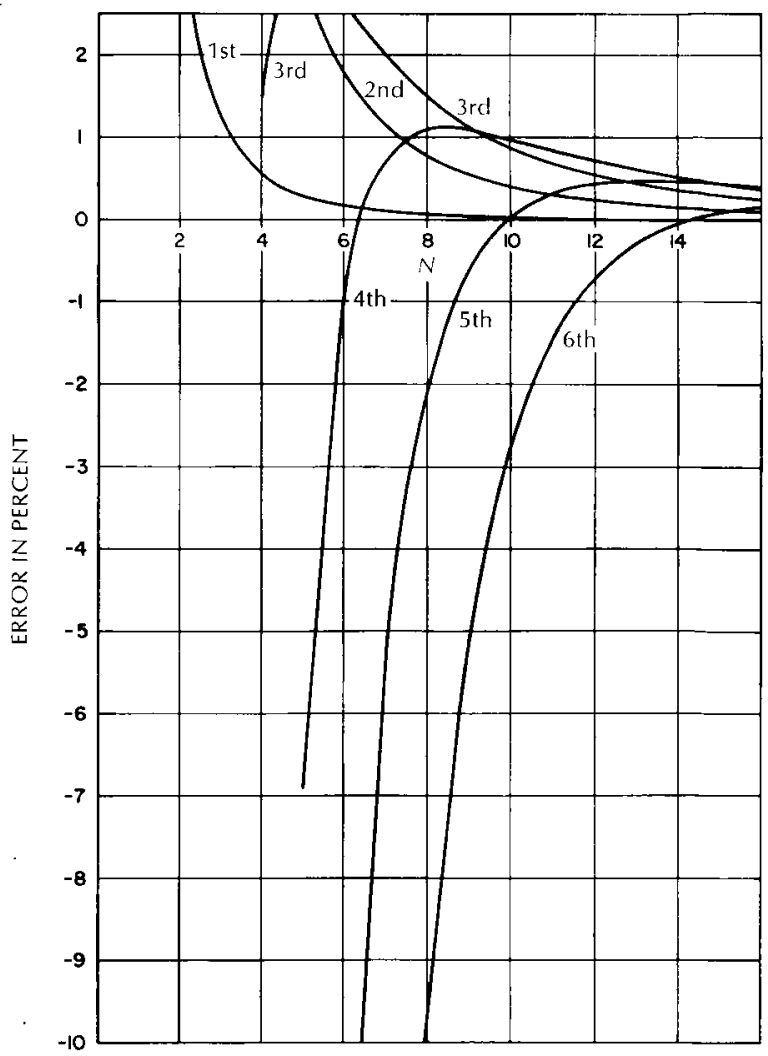

Graph 8-Per Cent Error in Mode Frequency for a Cantilever Beam, Passive Circuit 
These error curves are plotted in Graph 9. It should be noted that the calculated frequencies are always too low. As $N$ becomes sufficiently large, the error decreases inversely as $N^{4}$. Specifically, it can be shown that the first error term corresponds to a fractional error in frequency

$$
\varepsilon=-\frac{3 \pi^{4}}{320}\left(\frac{i}{N}\right)^{4}
$$

where $i$ is the mode number. The correct frequency for the corresponding continuous uniform pinned beam of unit length is $(i \pi)^{2}$.

When one compares the frequencies of a pinned beam with those for a cantilever or built-in beam, the frequencies are found to alternate. In fact, for large $i$, the pinned frequencies occur near the midpoint between adjacent frequencies for the cantilever or built-in beams. Thus if the only error were due to the basic approximation of (14), one would expect the error curves for the cantilever beam to alternate with those for the pinned beam and run roughly parallel to them. Instead, one finds that all three boundary condition methods result in a shift upward of the error curves, resulting in an improvement in accuracy for large $i / \mathrm{N}$ ratios in particular. This effect is most pronounced for the passive circuit method.

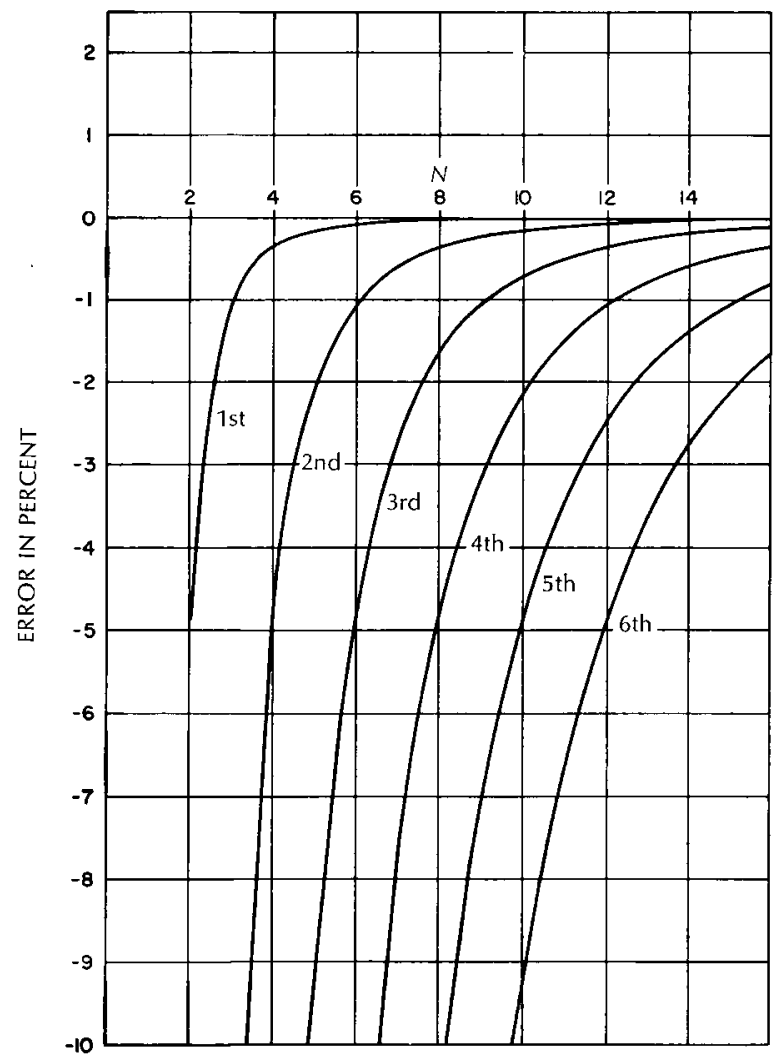

Graph 9 - Per Cent Error in Mode Frequency for a Pinned Beam, $h^{4}$ Approximation

\section{CONCLUSIONS}

1. The use of higher-order differences enables one to obtain improved accuracy with essentially no increase in the required number of amplifiers, if analog computers are used, or in the computation time, if digital computers are used.

2. The representation of boundary conditions by using one-sided differences of order $h^{4}$ is best suited to relatively small $i / N$ ratios, where $i$ is the mode number and $N$ is the total number of cells, corresponding to accuracies of at least 0.25 per cent.

3 . The use of symmetry at the boundaries is most useful for small $N(N \leq 9$, for example) where accuracies of the order of 1 per cent can be obtained for all but the highest two or three modes.

4. The passive-circuit approach is quite accurate in general, and, in particular, for large $i / N$ ratios, i.e., for obtaining the higher modes using relatively few cells.

5. The passive-circuit method gives modes that are orthogonal. The symmetry method results in nearly orthogonal modes, but the $h^{4}$ method produces modes with significant deviations from orthogonality.

6. For practical numbers of cells, boundary condition representation by a strict $h^{4}$ method may be less desirable than the use of simpler methods which require fewer cells for 1 per cent accuracy in mode frequency.

\section{BIBLIOGRAPHY}

1. R. M. Howe and V. S. Haneman, The Solution of Partial Differential Equations by Difference Methods Using the Electronic Differential Analyzer, Proc. IRE, vol. 41, pp. 1497-1508; October, 1953.

2. G. D. McCann and R. H. MacNeal, Beam Vibration Analysis with the Electric Analog Computer, J. Appl. Mech., vol. 17, pp. 13-26; 1950.

3. M. E. Fisher, Higher Order Differences in the Analogue Solution of Partial Differential Equations, J. ACM, vol. 3, pp. 325347; October, 1956.

4. M. E. Fisher, Limitations Due to Noise, Stability, and Component Tolerance on the Solution of Partial Differential Equations by Differential Analyzers, J. Electronics and Control, vol. 8, pp. 113-126; February, 1960.

5. C. E. Howe and R. M. Howe, Application of Difference Techniques to the Lateral Vibration of Beams Using the Electronic Differential Analyzer, Engrg. Res. Inst., University of Michigan, Ann Arbor, Rept. No. AIR-7; February, 1954.

6. R. W. Michie, Frequency Errors in Analog Computer Methods, Lockheed Aircraft Corp., Burbank, Calif., Rept. No. MAS-46; 1956. 

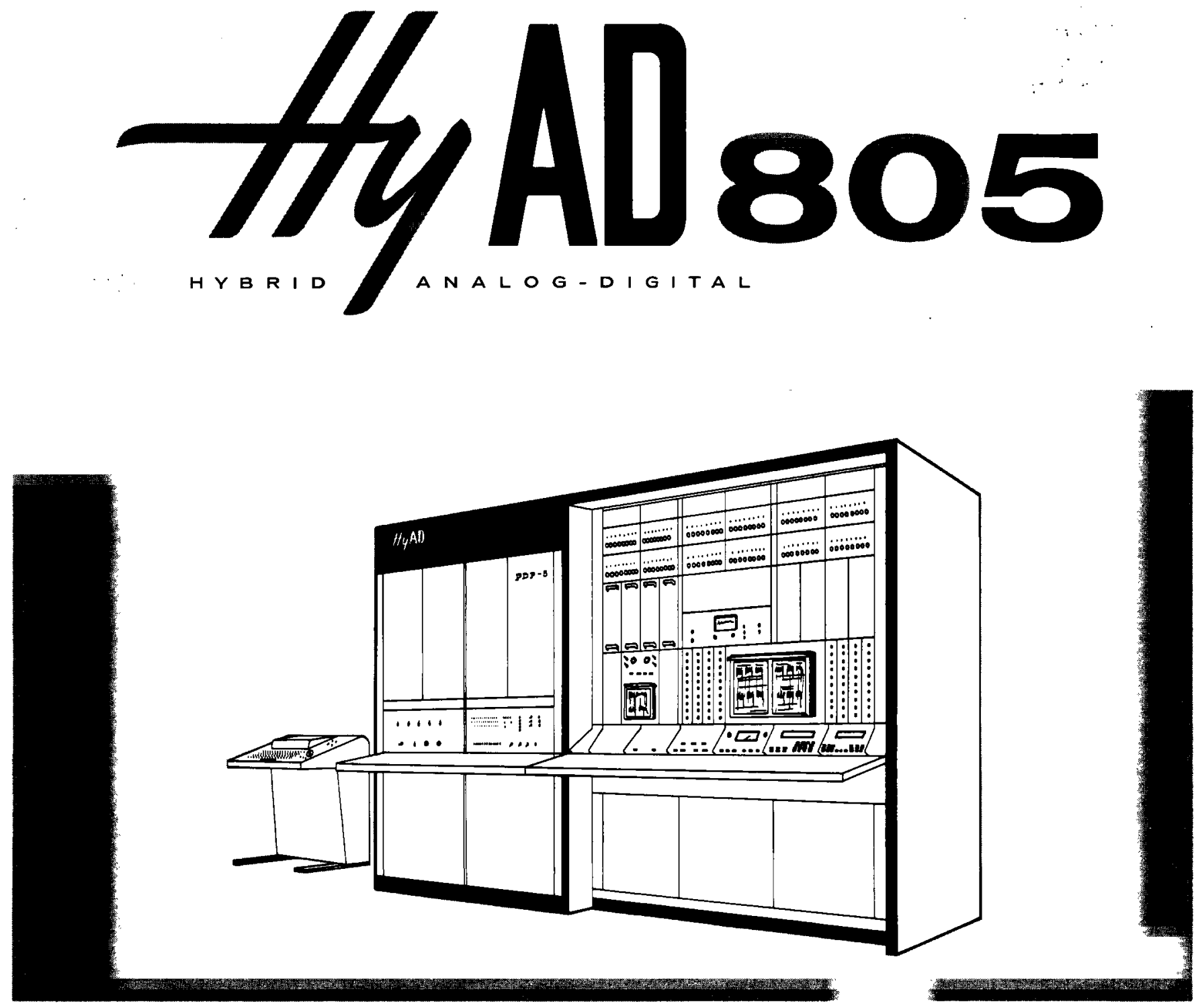

\section{The practical way to get into hybrid computation}

\section{Start with the $\$ 98,000$ basic system}

32 amplifiers

12 integrators

40 potentiometers

5 non-linear devices

5 hybrid devices

1 patchable logic system, half-expanded

1 PDP-5 with 4K core memory, typewriter, punch and reader

814 bit A-D channels

814 bit D-A channels

1 conversion and mode control system
Expand later to the complete system

80 amplifiers

24 integrators

80 servo-set potentiometers

20 non-linear devices

24 hybrid devices

1 fully expanded patchable logic system

1 PDP.5 with $32 \mathrm{~K}$ core memory, typewriter, and high speed punch and reader

1614 bit A-D channels

1614 bit $D$-A channels

1 conversion and mode control system
The HyAD 805 software package includes the $A D$ SPOUSE (stored program output setup equipment) for complete analog computer setup and control, standard PDP-5 routines and double precision routines for common operations such as sine, cosine, square root, binary to decimal conversion, interpretive floating point, etc.

Additional AD-80 consoles expand the system for larger problems or may be time-shared for solving separate problems.

The SPOUSE program with a PDP-5 and interface serves as the standard input-output unit for the larger AD-256 bi-polar analog computers. Other larger digital computers are easily interfaced with either the AD-80 or the AD-256. Consider these integrated HyAD systems for your hybrid requirements.
Write to Applied Dynamics for additional information or for a personal visit by an AD Applications Engineer.

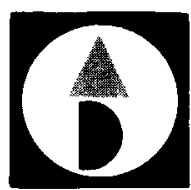

APP L I E D 


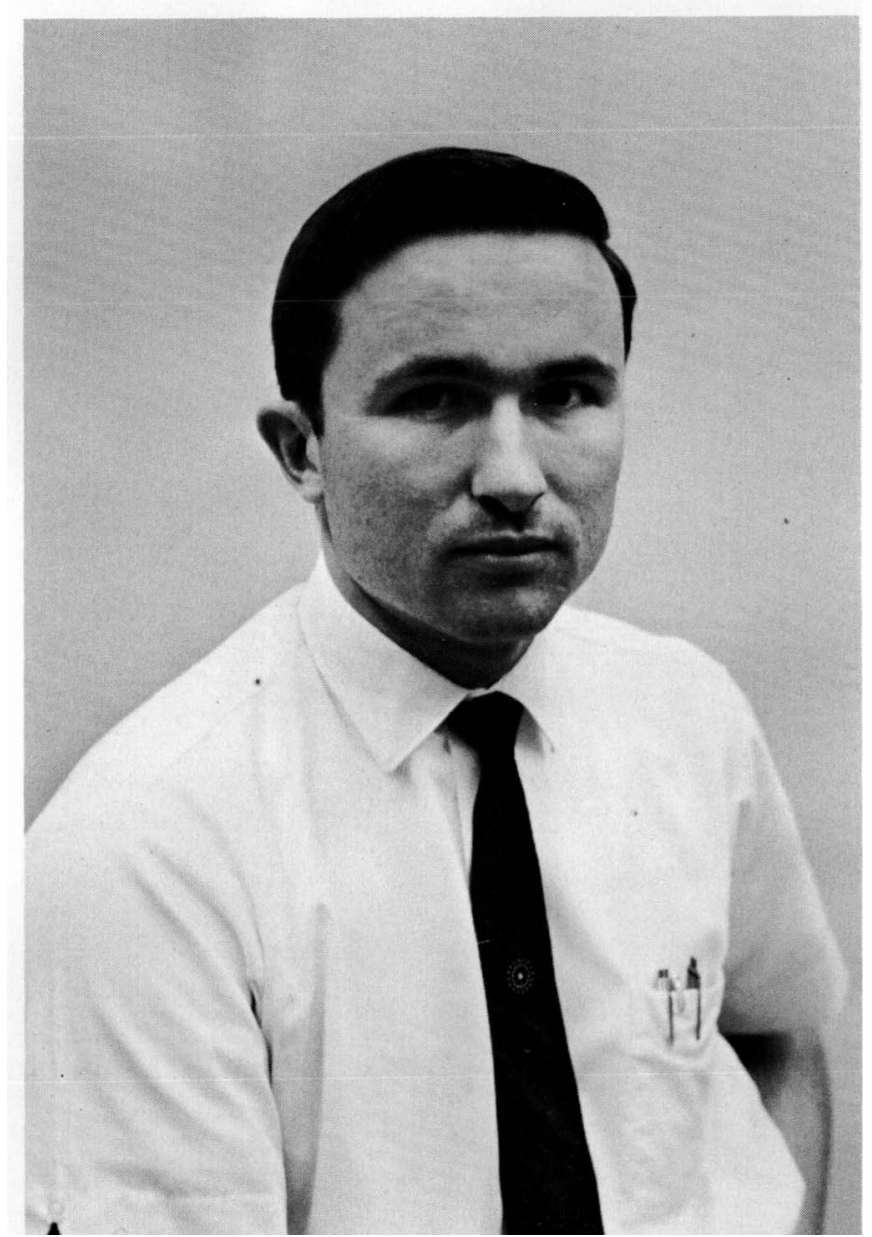

RICHARD J. MCGRATH was born in Milwaukee, Wisconsin. He served with the U.S. Army from 1951 through 1953 and worked summers with General Electric and the Wisconsin Telephone Company. He received his $\mathrm{BSEE}, \mathrm{MS}$, and $\mathrm{PhD}$, all from the University of Wisconsin, then served as assistant professor at Wisconsin, mainly carrying on research in the area of adaptive systems. He then joined the Aerospace Corporation, El Segundo, California, where he worked on satellite attitude control and terminal guidance problems. At present he is at Aerospace's San Bernardino Operations in the Guidance and Control Department. His interests lie in the applications of information theory and computing with emphasis on control and data handling problems.

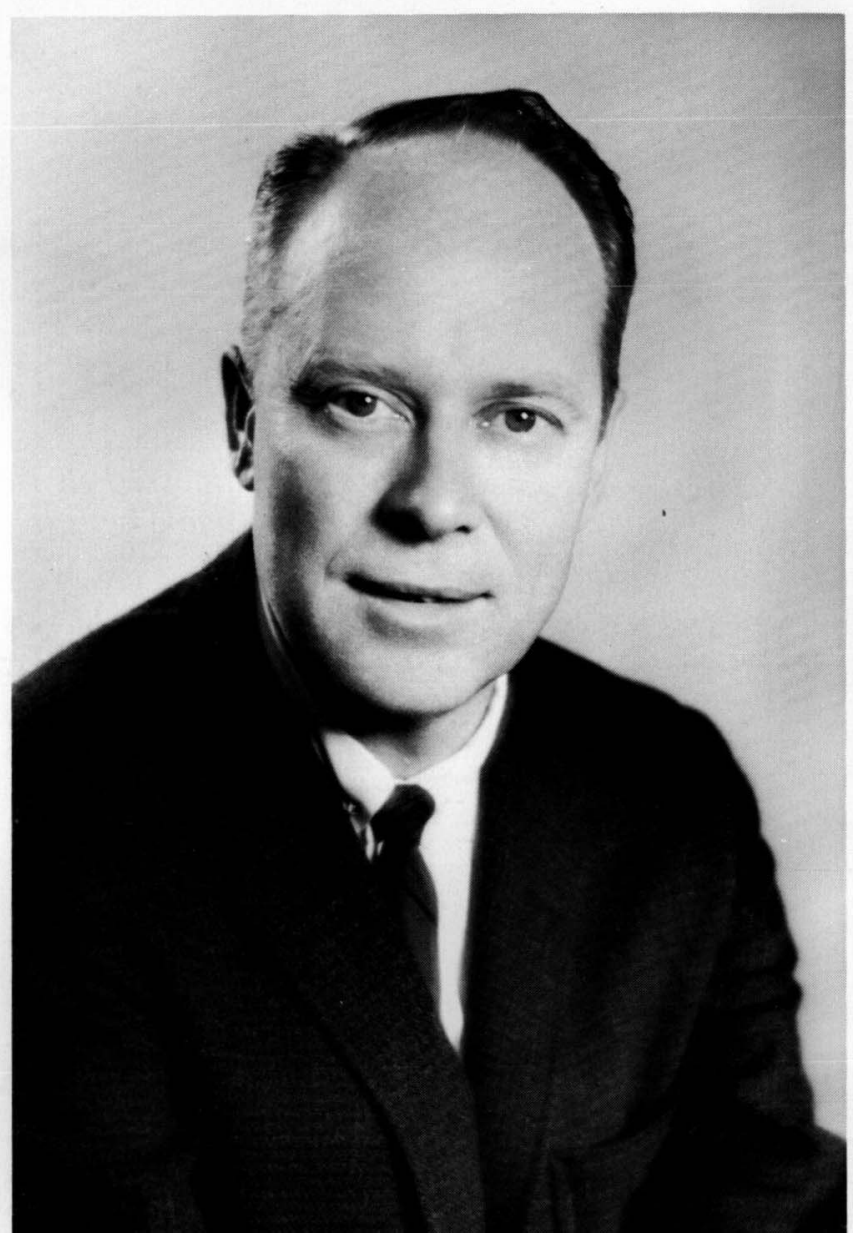

VINCENT C. RIDEOUT is Professor of Electrical Engineering at the University of Wisconsin, where he has been on the staff since 1946. At Wisconsin his principal research interests have been in computer simulation, particularly as applied to the study of nonlinear systems with random inputs. He has pursued similar interests during two years on leave-of-absence from Wisconsin, at the Indian Institute of Science, Bangalore (1954-55), and at the University of Colorado (1963-64). Recently Professor Rideout has concerned himself with problems relating to hybrid computation, and digital simulation of hybrid computers. He consults in connection with computing and control problems for a number of industrial firms and laboratories. He is the author of a number of papers, a section of the Computer Handbook (Huskey and Korn, Editors), and a senior-level textbook. 